\title{
Crescimento inicial de arbóreas nativas em solo salino-sódico do nordeste brasileiro tratado com corretivos
}

\author{
Francisco Vanies da Silva Sál, Josinaldo Lopes Araujo², Maurício Cavalcante de Novaes ${ }^{1}$, \\ Alexandre Paiva da Silva ${ }^{3}$,Francisco Hevilásio Freire Pereira ${ }^{3}$, Kilson Pinheiro Lopes ${ }^{3}$
}

\begin{abstract}
RESUMO
O excesso de sais e de sódio no solo é um dos fatores que mais contribuem para a degradação química dos solos de perímetros irrigados, em regiões áridas e semiáridas. Por essa razão, objetivou-se, com este trabalho, avaliar o efeito de corretivos, na recuperação de um solo degradado por excesso de sais e sódio, e o crescimento inicial de cinco arbóreas nativas do bioma Caatinga, em solo salino-sódico. Dois experimentos foram conduzidos, em casa de vegetação, no CCTA/UFCG, utilizando-se amostras de um solo salino-sódico. O primeiro experimento constou de cinco tratamentos de correção do solo: sem corretivo, gesso agrícola na dose de $100 \%$ da necessidade de gesso (NG), gesso agrícola na dose de 50\% NG mais matéria orgânica (MO), enxofre elementar (S) na dose de $100 \%$ NG e S na dose de $50 \%$ $\mathrm{NG}+\mathrm{MO}$, com 15 repetições. No segundo experimento, os tratamentos foram constituídos por um esquema fatorial 5 x 5, sendo cinco espécies arbóreas: tamboril (Enterolobium contortisiliquum), sabiá (Mimosa caelsalpiniifolia), jurema-preta (Mimosa tenuiflora), craibeira (Tabebuia aurea) e pereiro (Aspidosperma pyrifolium) e cinco tratamentos referentes à correção do solo do primeiro experimento, com três repetições. A aplicação de gesso agrícola ou S com ou sem MO melhorou quimicamente o solo salino-sódico estudado, especialmente diminuindo a PST. Estes tratamentos proporcionaram incrementos no crescimento e acúmulo de massa de matéria seca das espécies arbóreas, principalmente jurema-preta, sabiá e tamboril, e diminuíram o estresse provocado pelo solo salino-sódico sobre as plantas, aumentando a taxa fotossintética.
\end{abstract}

Palavras-chave: degradação ambiental, gesso agrícola, corretivos da salinidade, enxofre elementar.

\section{ABSTRACT \\ Initial growth of native trees on saline-sodic soil from northeastern Brazil using amendments}

Salts and sodium in excess on soil is one of the main factors that contribute to chemical degradation of soils from arid and semiarid irrigated areas. The objective of this work was to evaluate the soil amendments effect on the recovery of a soil degraded by salts and sodium in excess and on the initial growth of five native trees of Caatinga biome on saline-sodic soil. Two experiments were carried out in a greenhouse at CCTA/UFCG, using samples of a saline-sodic soil. The first experiment consisted of five soil amendment treatments: without correction, agricultural gypsum at the rate of $100 \%$ of the gypsum need (NG), agricultural gypsum at the rate of 50\% NG + organic matter (OM), elementary sulfur (S) at the rate of $100 \% \mathrm{NG}$ and elementary sulfur at the rate of 50\% NG + OM, with 15 replicates. In the second experiment, the treatments were conducted in a factorial scheme 5 x 5 , with five native trees species [tamboril (Enterolobium contortisiliquum), sabiá (Mimosa caelsalpiniifolia), jurema-preta (Mimosa tenuiflora), craibeira (Tabebuia aurea) e pereiro (Aspidosperma pyrifolium) and with the five soil amendment treatments from the first

Recebido para publicação em 17/08/2012 e aprovado em 08/03/2013.

${ }^{1}$ Graduandos em Agronomia. Centro de Ciências e Tecnologia Agroalimentar, Universidade Federal de Campina Grande, Campus Pombal, Rua Jairo Feitosa, 1770, Bairro dos Pereiros, 58840-000, Pombal, Paraíba, Brasil.vanies_agronomia@ hotmail.com; mauro.novaes@hotmail.com

${ }^{2}$ Engenheiro Florestal, Doutor. Centro de Ciências e Tecnologia Agroalimentar, Universidade Federal de Campina Grande, Campus Pombal, Rua Jairo Feitosa, 1770, Bairro dos Pereiros, 58840-000, Pombal, Paraíba, Brasil. jhosinal_araujo@yahoo.com.br (autor para correspondência).

${ }^{3}$ Engenheiros-Agrônomos, Doutores. Centro de Ciências e Tecnologia Agroalimentar, Universidade Federal de Campina Grande, Campus Pombal, Rua Jairo Feitosa, 1770, Bairro dos Pereiros, 58840-000, Pombal, Paraíba, Brasil. paivadasilva@gmail.com, fhfpereira@hotmail.com; kilson@ccta.ufcg.edu.br 
experiment, with three replicates. The use of gypsum or elementary sulfur with or without organic matter improved chemically the saline-sodic soil studied, particularly by decreasing the percentage of exchangeable sodium. These treatments promoted increases on growth and on dry matter accumulation of native trees, especially jurema-preta, sabiá and tamboril and decreased the stress caused by saline-sodic soil on plants by increasing the photosynthetic rate.

Key words: environmental degradation, agricultural gypsum, salinity amendments, elementary sulfur.

\section{INTRODUÇÃO}

Os solos com excesso de sais e, ou, de sódio estão distribuídos em todo o mundo, especialmente em regiões áridas e semiáridas do globo, onde a evapotranspiração supera a precipitação pluviométrica (Zaka et al., 2003; Qadir et al., 2007). No Brasil, embora, de maneira geral, sejam de pouca ocorrência, na região semiárida do nordeste a degradação química desses solos causa redução significativa da produção agrícola em perímetros irrigados, levando ao abandono de extensas áreas (Ribeiro et al., 2003).

A salinidade causa alterações químicas e físicas no solo, as quais, em última instância, afetam o comportamento das espécies vegetais neles cultivadas (Hasegawa et al., 2000; Munns, 2002). As alterações químicas afetam a fertilidade do solo, promovendo elevados teores de ânions, como cloretos, sulfatos, carbonatos, bicarbonatos e boratos na solução solo, os quais podem causar distúrbios nutricionais nas plantas (Santos \& Muraoka, 1997; Duran et al., 2000). Sob condições de sodicidade, outro agravante é o elevado $\mathrm{pH}$ (em geral, superior a 8,5), o que origina toxidez de íons, como o sódio e o cloro, ou deficiências nutricionais, especialmente de micronutrientes catiônicos, como o zinco, cobre, manganês e ferro (Raij, 1991). Os íons $\mathrm{Na}^{+}$, em solos sódicos ou salinosódicos, promovem o deslocamento dos cátions $\mathrm{Mg}^{2+}$, $\mathrm{Ca}^{2+} \mathrm{e} \mathrm{K}^{+}$, substituindo-os no complexo de troca, diminuindo sua disponibilidade para as plantas, e promovendo dispersão de argilas, uma vez que os cátions bivalentes são substituídos por monovalentes, promovendo o aumento da espessura da dupla camada difusa (McBride, 1995).

Além das alterações nutricionais, nas plantas, o excesso de sais e de sódio promove alterações fisiológicas, como a diminuição da taxa fotossintética, condutância estomática, transpiração e condutividade hidráulica das raízes, além de injúrias e abscisão foliar, levando à redução da taxa de crescimento (Romero-Aranda et al., 1998; Munns, 2002). Além disso, a dispersão de argilas pelo excesso de sódio promove o entupimento dos poros do solo, diminuindo a água disponível às plantas e, com isso, afetando as trocas gasosas das plantas (Romero-Aranda et al., 1998).
Na recuperação desses solos, a aplicação de condicionadores do solo, como o gesso agrícola, o enxofre elementar, o ácido sulfúrico e os resíduos orgânicos, tem sido a prática mais recomendada. Os efeitos positivos do uso desses produtos na melhoria dos atributos químicos e físicos do solo têm sido verificados em muitos trabalhos (Vital et al., 2005; El-Dardiry, 2007; Leite et al., 2007; Mohamed et al., 2007; Stamford et al., 2007; Zia et al., 2007; Gill et al., 2008; Leal et al., 2008; Melo et al., 2008; Pazhanivelan et al., 2008; Gharaibeh et al., 2009; Gill et al., 2009). Dentre esses corretivos, o gesso agrícola tem sido o mais recomendado, por seu baixo custo e sua maior disponibilidade no mercado (Leite et al., 2007).

Além do uso de corretivos, o cultivo de espécies mais tolerantes à salinidade é uma das medidas que podem acelerar o processo de recuperação desses solos, reintegrando as áreas salinizadas mais rapidamente à produção agrícola (Qadir et al., 1996). As espécies arbóreas têm, como vantagem, o sistema radicular profundo, aumentando a permeabilidade do solo, a lixiviação dos sais e o abaixamento do lençol freático (Qadir et al., 1996). Além disso, geralmente são espécies de uso múltiplo, fixam o nitrogênio atmosférico e concentram grande quantidade de matéria orgânica no solo. Contudo, poucos trabalhos foram realizados, avaliando a tolerância à salinidade dessas espécies, principalmente as arbóreas de ocorrência em ecossistema de Caatinga (Tertuliano \& Santos, 1998; Holanda et al., 2007; Freitas et al., 2010; Sousa et al., 2012). Portanto, a principal hipótese deste trabalho é que o uso de corretivos/condicionadores da salinidade do solo, estabelece condições mais adequadas ao crescimento de espécies nativas, nesses solos.

Por essa razão, objetivou-se, neste trabalho, avaliar o efeito de corretivos na recuperação de um solo degradado por excesso de sais e sódio e o crescimento inicial de cinco espécies arbóreas, nativas do bioma Caatinga, em solo salino-sódico.

\section{MATERIAL E MÉTODOS}

\section{Local e condução dos experimentos}

Dois experimentos foram conduzidos, em casa de vegetação do Centro de Ciências e Tecnologia Agroalimentar (CCTA), da Universidade Federal de Campina Grande 
(UFCG), Campus de Pombal, Paraíba, utilizando-se amostras de um solo salino-sódico. As amostras foram obtidas aleatoriamente, na profundidade de $0-40 \mathrm{~cm}$, em lote pertencente ao Setor de Fruticultura do Instituto Federal de Educação, Ciência e Tecnologia da Paraíba (IFPB-PB), localizado no Perímetro Irrigado de São Gonçalo, a 10 km do município de Sousa.

Após secadas ao ar, destorroadas e passadas em peneira de malha de 2,0 $\mathrm{mm}$, as amostras foram encaminhadas ao Laboratório de Solos e Nutrição de Plantas do CCTA/UFCG para sua caracterização química e física (Tabela 1), de acordo com metodologia proposta pela Embrapa (1997). Visando à sua classificação quanto à salinidade, foram avaliados os valores de pHes, CEes (condutividade elétrica do extrato de saturação), os teores solúveis de $\mathrm{Ca}, \mathrm{Mg}, \mathrm{K}$ e Na e PST (Percentagem de Sódio Trocável) conforme Richards (1954).

\section{Experimento 1}

O delineamento foi inteiramente casualizado (DIC), com cinco tratamentos de correção do solo: sem corretivo, gesso agrícola na dose de $100 \%$ da necessidade de gesso (NG), gesso agrícola na dose de $50 \%$ NG mais matéria orgânica (MO), enxofre elementar (S) na dose de $100 \%$ NG e S na dose de $50 \%$ NG mais MO, com 15 repetições, totalizando 75 vasos com $6 \mathrm{dm}^{3} \mathrm{de}$ solo.

A dose de gesso agrícola correspondente à NG foi calculada com base no teor de sódio trocável do solo, visando a diminuir sua PST para $15 \%$. Para o S, foi empregado produto analítico (pa), cuja dose foi calculada com base na dose de gesso recomendada, objetivando fornecer a mesma quantidade de enxofre fornecida por via do gesso. Para a MO, foi utilizado o esterco bovino curtido, na dose correspondente a $15 \%$ do volume de solo.

Após a aplicação dos corretivos, os solos foram mantidos por 30 dias com umidade correspondente a $70 \%$ da capacidade de campo. A fase seguinte correspondeu à lavagem dos solos, aplicando-se um volume de água (CE $<0,25 \mathrm{dSm}^{-1}$ ), equivalente a duas vezes a porosidade total do solo. Após a lavagem, foram retirados de cada vaso cerca de $300 \mathrm{~g}$ de solo, com os quais foi realizada uma nova caracterização química, conforme os procedimentos anteriormente descritos.

\section{Experimento 2}

No segundo experimento, também em DIC, os tratamentos foram arranjados em esquema fatorial $5 \times 5$, sendo cinco espécies arbóreas: tamboril (Enterolobium contortisiliquum (Vell.) Morong), sabiá (Mimosa caelsalpiniifolia Benth.), jurema-preta (Mimosa tenuiflora (Willd.) Poir.), craibeira (Tabebuia aurea (Silva Manso) Benth. \& Hook. f. ex S. Moore) e pereiro (Aspidosperma pyrifolium Mart.) e cinco tratamentos referentes à correção do solo do primeiro experimento (sem corretivo, gesso agrícola na dose de $100 \%$ da $\mathrm{NG}$, gesso agrícola na dose de $50 \% \mathrm{NG}+\mathrm{MO}, \mathrm{S}$ na dose de $100 \% \mathrm{NG}$ e $\mathrm{S}$ na dose de $50 \% \mathrm{NG}+\mathrm{MO}$ ), com três repetições, totalizando 75 vasos com $6 \mathrm{dm}^{3}$ de solo.

As espécies arbóreas empregadas foram escolhidas de acordo com o seu potencial de utilização em reflorestamentos e em recuperação de áreas degradadas e pela im-

Tabela 1. Atributos químicos e físicos do solo salino-sódico utilizado no experimento

\begin{tabular}{|c|c|c|c|}
\hline Atributos químicos & Valor & Atributos físicos & Valor \\
\hline Complexo sortivo & & Areia $\left(\mathrm{g} \mathrm{kg}^{-1}\right)$ & 594 \\
\hline $\mathrm{pH}\left(\mathrm{CaCl}_{2}\right)$ & 9,8 & Silte $\left(\mathrm{g} \mathrm{kg}^{-1}\right)$ & 305 \\
\hline $\mathrm{P}\left(\mathrm{mg} \mathrm{kg}^{-1}\right)$ & 5,9 & Argila $\left(\mathrm{g} \mathrm{kg}^{-1}\right)$ & 101 \\
\hline $\mathrm{K}^{+}\left(\mathrm{cmol}_{\mathrm{c}} \mathrm{dm}^{-3}\right)$ & 0,7 & Ds $\left(\mathrm{g} \mathrm{cm}^{-3}\right)$ & 1,45 \\
\hline $\mathrm{Na}^{+}\left(\mathrm{cmol}_{\mathrm{c}} \mathrm{dm}^{-3}\right)$ & 10,3 & $\mathrm{Dp}\left(\mathrm{g} \mathrm{cm}^{-3}\right)$ & 2,81 \\
\hline $\mathrm{Ca}^{2+}\left(\mathrm{cmol}_{\mathrm{c}} \mathrm{dm}^{-3}\right)$ & 1,0 & & \\
\hline $\mathrm{Mg}^{2+}\left(\mathrm{cmol}_{\mathrm{c}} \mathrm{dm}^{-3}\right)$ & 1,1 & Umidade $\left(\mathrm{g} \mathrm{kg}^{-1}\right)$ & \\
\hline $\mathrm{H}+\mathrm{Al}\left(\mathrm{cmol}_{\mathrm{c}} \mathrm{dm}^{-3}\right)$ & 0,0 & 0,010 MPa & 160 \\
\hline $\mathrm{SB}\left(\mathrm{cmol}_{\mathrm{c}} \mathrm{dm}^{-3}\right)$ & 13,2 & $0,033 \mathrm{MPa}$ & 117 \\
\hline CTC $\left(\mathrm{cmol}_{\mathrm{c}} \mathrm{dm}^{-3}\right)$ & 13,2 & $1,500 \mathrm{MPa}$ & 80 \\
\hline $\mathrm{V}(\%)$ & 100,0 & & \\
\hline PST $(\%)$ & 78,7 & & \\
\hline \multicolumn{4}{|l|}{ Extrato de saturação } \\
\hline CEes $\left(\mathrm{dSm}^{-1}\right)$ & 39,9 & & \\
\hline $\mathrm{pH}$ & 9,4 & & \\
\hline $\mathrm{Na}^{+}\left(\operatorname{mmol}_{\mathrm{c}} \mathrm{L}^{-1}\right)$ & 289,7 & & \\
\hline $\mathrm{Ca}^{2+}+\mathrm{Mg}^{2+}\left(\mathrm{mmol}_{\mathrm{c}} \mathrm{L}^{-1}\right)$ & 7,0 & & \\
\hline PST (\%) & 87,8 & & \\
\hline $\operatorname{RAS}\left(\mathrm{mmol}_{\mathrm{c}} \mathrm{L}^{-1}\right)^{0,5}$ & 489,7 & & \\
\hline
\end{tabular}

$\mathrm{P}, \mathrm{K}^{+}$e $\mathrm{Na}^{+}$: Extrator Mehlich-1; $\mathrm{H}^{+}+\mathrm{Al}^{+3}$ : Extrator acetato de $\mathrm{Ca}^{+2} 0,5 \mathrm{~mol} \mathrm{~L}^{-1} \mathrm{pH} 7 ; \mathrm{Al}^{+3}, \mathrm{Ca}^{+2}, \mathrm{Mg}^{+2}$ : Extrator $\mathrm{KCl}_{1 \mathrm{~mol} \mathrm{~L}}^{-1}$.

Rev. Ceres, Viçosa, v. 60, n.3, p. 388-396, mai/jun, 2013 
portância econômica que vêm mostrando na região. As sementes das espécies foram germinadas em tubetes de $300 \mathrm{~mL}$, preenchidos com substrato composto por solo de barranco e areia. Posteriormente, as plantas foram transplantadas para os vasos empregados no primeiro experimento, com os respectivos tratamentos, após terem atingido altura mínima de $15 \mathrm{~cm}$.

Após o transplantio das mudas, em todos os vasos foi realizada uma adubação básica com nitrogênio (250 $\mathrm{mg} \mathrm{dm}^{-3}$ ), fósforo (150 $\left.\mathrm{mg} \mathrm{dm}^{-3}\right)$, potássio $\left(100 \mathrm{mg} \mathrm{dm}^{-3}\right)$, magnésio $\left(20 \mathrm{mg} \mathrm{dm}^{-3}\right)$ e micronutrientes. Os macronutrientes foram fornecidos na forma de solução, utilizando-se as seguintes fontes pa: ureia $\left[\left(\mathrm{CO}\left(\mathrm{NH}_{2}\right)_{2}\right)\right.$, ácido fosfórico $\left(\mathrm{H}_{3} \mathrm{PO}_{4}\right)$, fosfato monobásico de potássio $\left(\mathrm{KH}_{2} \mathrm{PO}_{4}\right)$, cloreto de potássio $(\mathrm{KCl})$ e sulfato de magnésio $\left(\mathrm{MgSO}_{4} \cdot 7 \mathrm{H}_{2} \mathrm{O}\right)$. A adubação com micronutrientes consistiu na pulverização manual das plântulas com solução contendo sulfato de cobre $\left(1,0 \mathrm{~g} \mathrm{~L}^{-1}\right)$, cloreto de manganês $\left(2,0 \mathrm{~g} \mathrm{~L}^{-1}\right)$, sulfato ferroso $\left(5,0 \mathrm{~g} \mathrm{~L}^{-1}\right)$ e sulfato de zinco $(2,5$ $\mathrm{g} \mathrm{L}^{-1}$ ), usando fontes pa. $\mathrm{O}$ fornecimento de micronutrientes por via foliar foi realizado em duas etapas, ocorrendo a primeira cinco dias após o transplantio e, a segunda, trinta dias após.

Após 20 dias do transplantio, foram avaliadas nas plantas as taxa de transpiração e de fotossíntese, a condutância estomática e a concentração intercelular de $\mathrm{CO}_{2}$, utilizando-se o analisador de gás infravermelho - IRGA (Infrared Gas Analyzer) LI-COR 6400. Durante 90 dias após o transplantio das plântulas, foram realizadas medições quinzenais do diâmetro do caule e altura de planta, com os quais foram determinados os acréscimos de crescimento em diâmetro e em altura de cada espécie. Posteriormente, as partes aéreas foram separadas das raízes e secadas em estufa, a $65-70^{\circ} \mathrm{C}$, para a obtenção da matéria seca da parte aérea (MSPA), da matéria seca de raízes (MSR) e da matéria seca total (MST). Com os dados de matéria seca total, calculou-se a produção de matéria seca relativa total das espécies vegetais, empregando-se a seguinte expressão:

\section{MSRel $=($ MSCC $/$ MSSC $) \times 100$}

em que:

MSRel = matéria seca relativa;

MSCC $=$ matéria seca produzida num tratamento com corretivo;

MSSC = matéria seca produzida no tratamento sem corretivo.

Em ambos os experimentos, a análise estatística constou da análise de variância, do teste de médias (Tukey), utilizando-se o software SISVAR 4.0, e da análise de correlação linear simples entre variáveis, utilizando-se o software SAEG 9.0, a 5\% de significância.

\section{RESULTADOS E DISCUSSÃO}

\section{Experimento 1}

Os tratamentos de correção influenciaram os atributos do solo, tanto do complexo sortivo, quanto do extrato de saturação (Tabela 2). Observou-se que, independentemente do tipo de corretivo, houve diminuição dos valores de $\mathrm{pH}$, de sódio trocável e da percentagem de sódio trocável (PST) e aumento dos teores de cálcio e magnésio trocáveis.

A adição de gesso agrícola, na dose de $100 \%$ da necessidade de gesso (NG), ou a adição de $\mathrm{S}$, na dose correspondente a $100 \%$ da NG, foram os tratamentos que mais contribuíram para o abaixamento do $\mathrm{pH}$ do solo (Tabela 2). Os tratamentos com adição de gesso agrícola proporcionaram maior declínio nos teores de sódio $\left(\mathrm{Na}^{+}\right)$trocável e maior aumento nos de cálcio $\left(\mathrm{Ca}^{2+}\right)$ e de magnésio $\left(\mathrm{Mg}^{2+}\right)$ trocáveis (Tabelas 2). A aplicação do gesso agrícola a 100\% reduziu em $57,4 \%$ o teor de $\mathrm{Na}^{+}$trocável, em relação ao do solo salino sem corretivo.

A diminuição dos teores trocáveis de $\mathrm{Na}^{+}$pela aplicação do sulfato de cálcio, também observada em vários trabalhos (Leite et al., 2007; Leal et al., 2008; Sousa et al., 2012), é decorrente, principalmente, do deslocamento do sódio do complexo de troca pelo cálcio liberado pelo corretivo. Neste processo, o sódio é deslocado pelo $\mathrm{Ca}^{+2} \mathrm{e}$ pelo $\mathrm{Mg}^{+2}$ para a solução do solo, para reagir com os ânions sulfatos, formando sulfato de sódio, o qual é removido da solução do solo, após a aplicação de uma lâmina de água. Assim, o sulfato de cálcio favoreceu a lixiviação de sódio e de íons $\mathrm{HCO}_{3}{ }_{3}^{-}$contribuindo, também, para a diminuição do $\mathrm{pH}$ do solo (Leite et al., 2007). Quanto ao enxofre, seu efeito no abaixamento do $\mathrm{pH}$ do solo salino deve-se, provavelmente, à sua oxidação biológica, a qual gera ácido sulfúrico no solo (Stamford et al., 2002), o que contribui para aumentar a solubilização de minerais contendo magnésio e cálcio, elevando assim os teores trocáveis desses elementos e diminuindo os teores de sódio no solo.

Os tratamentos com MO foram menos eficientes que os demais tratamentos na correção do solo salino-sódico; entretanto, em relação aos demais, proporcionaram aumentos nos teores de fósforo e potássio, decorrentes, provavelmente, do seu processo de mineralização.

O comportamento dos atributos químicos do solo do extrato de saturação em parte se assemelha àqueles obtidos no complexo sortivo (Tabela 2). Assim, a aplicação de corretivos ao solo proporcionou diminuição do $\mathrm{pH}$ e aumento dos teores solúveis de cálcio e magnésio. Por outro lado, apenas o tratamento com $100 \%$ da NG proporcionou redução significativa da PST. Verificou-se, ainda, que, após a aplicação da lâmina de lixiviação dos sais, houve diminuição de mais de $80 \%$ da salinidade do solo, 
medida pela CEes, em relação aos valores iniciais (Tabela 1). No tratamento sem corretivo (Tabela 2), a simples prática da lavagem reduziu para níveis baixos a salinidade do solo. Especificamente para o solo estudado neste trabalho, isso ocorreu, provavelmente, por causa de seu elevado teor de areia e baixo teor de argila (Tabela 1), proporcionando uma macroporosidade elevada, a qual facilita o processo de lixiviação dos sais. Contudo, é importante ressaltar que, em condições de campo, essa redução da CEes com a simples lavagem poderá não ocorrer, a menos que seja realizado o processo de aração profunda do solo, promovendo seu revolvimento, pois, mesmo em baixa proporção, a argila presente nesse solo, encontra-se dispersa (Sousa et al., 2012).

Embora a salinidade elevada provoque estresse hídrico nas plantas, diminuindo a disponibilidade de água pelo efeito osmótico (Munns, 2002), os elevados teores de $\mathrm{Na}^{+}$ no solo podem ser tanto ou mais prejudiciais, às plantas, quanto a salinidade elevada. Assim, a lavagem, à exceção do tratamento $100 \%$ NG, foi suficiente para reduzir a PST para valores considerados adequados à maioria das plantas $(<15 \%)$, conforme Richards (1954). A aplicação dos corretivos, contudo, diminuiu consideravelmente os valores dessa variável, principalmente a adição de gesso agrícola na dose de $100 \%$ da NG.

\section{Experimento 2}

Independentemente dos tratamentos de correção do solo, as arbóreas com maior incremento em altura, durante o período de avaliação, foram jurema-preta, sabiá e tamboril (Tabela 3). Em relação aos tratamentos de correção do solo, em geral, a aplicação de corretivos proporcionou aumentos significativos no incremento em altura das plantas, em relação ao tratamento controle, embora, com efeito não significativo em alguns casos (Tabela 3). Comparando-se apenas os tratamentos com corretivos, para as arbóreas jurema-preta, sabiá e tamboril, o crescimento em altura foi semelhante entre os tratamentos, com exceção do tamboril, que teve maior crescimento nos tratamentos com MO. Para craibeira e pereiro, não se observou um padrão definido de respostas no crescimento (Tabela 3). Para o crescimento em diâmetro, no tratamento sem corretivo, não houve diferença entre as arbóreas, enquanto nos demais tratamentos, a resposta foi variável, embora, de maneira geral, o pereiro tenha apresentado o menor crescimento em diâmetro (Tabela 3). Comparando-se os tratamentos de correção do solo, para cada arbórea, não foi observado efeito dos tratamentos em pereiro, juremapreta e sabiá, enquanto, nas demais arbóreas, a aplicação de $\mathrm{S}$ ou de gesso agrícola, ambos com ou sem MO, favoreceu o crescimento em diâmetro das plantas (Tabela 3).

Em relação à produção de matéria seca, a aplicação de corretivos, em geral, proporcionou incrementos significativos na produção de matéria seca da parte aérea e das raízes nas arbóreas jurema-preta, sabiá e tamboril, mas não afetou estas variáveis em craibeira e pereiro (Tabela 4). No caso da craibeira, isso indica uma maior tolerância ao estresse salino, uma vez que essa planta obteve produção de matéria seca semelhante à das arbóreas com maior valor para essa variável. Por outro lado, em pereiro, esse efeito indica alta sensibilidade à salinidade, tendo em vista que foi essa planta que apresentou os menores

Tabela 2. Atributos químicos do complexo sortivo e do extrato de saturação do solo, em função dos tratamentos após aplicação dos corretivos e lavagem

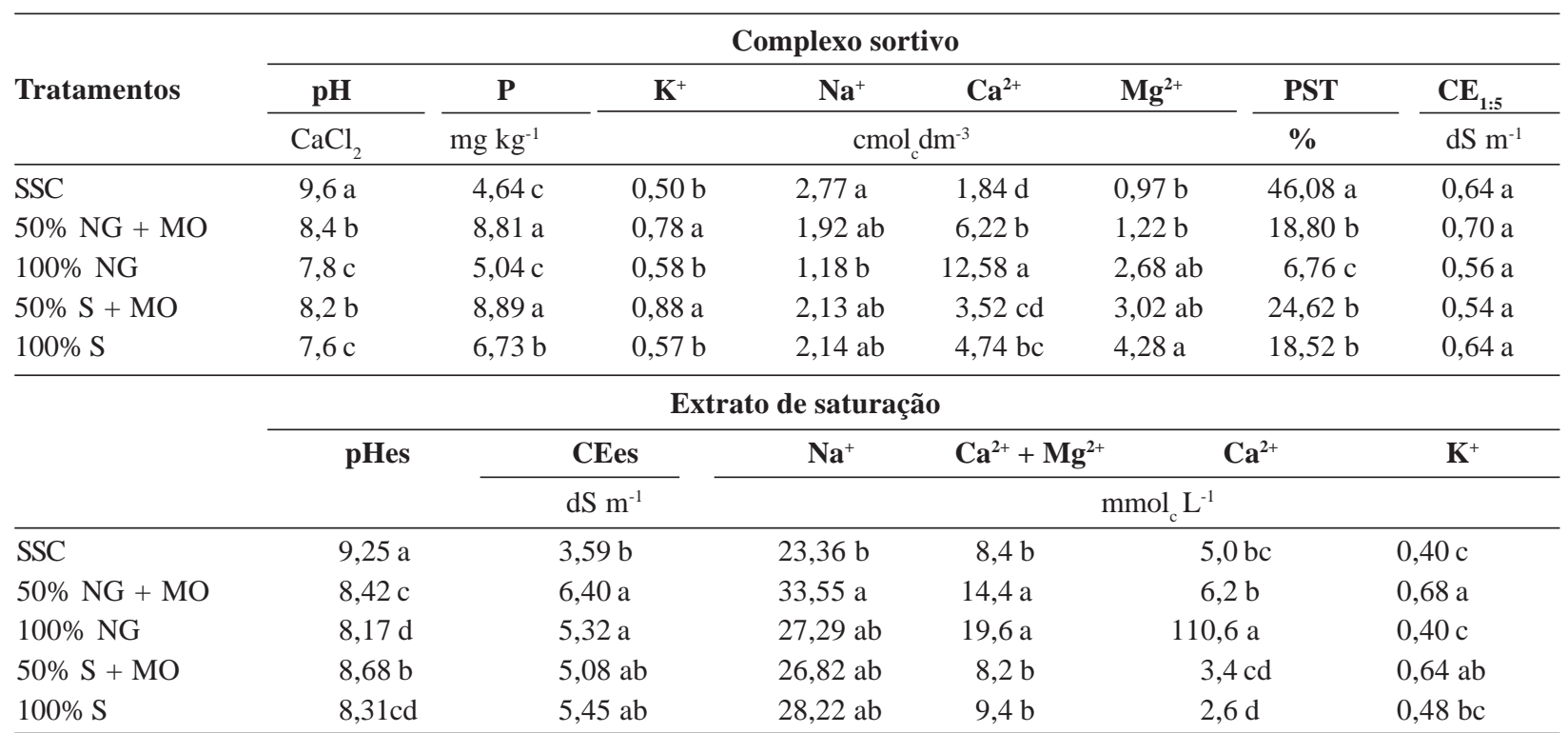

SSC: Solo sem corretivo; NG: Necessidade de gesso; MO: Matéria orgânica na dose de 15\% (v/v); S: enxofre elementar; CEes: Condutividade elétrica do extrato de saturação; PST: percentagem de sódio trocável; RAS: relação de adsorção de sódio. Médias seguidas de letras iguais, nas colunas, não diferem entre si (Tukey a 5\%).

Rev. Ceres, Viçosa, v. 60, n.3, p. 388-396, mai/jun, 2013 
valores para produção de matéria seca. Holanda et al. (2007) avaliaram a tolerância à salinidade de diversas espécies arbóreas, de ocorrência na Caatinga, e observaram que pereiro e craibeira foram as que obtiveram a menor produção de matéria seca da parte aérea, independentemente dos tratamentos avaliados.

Entre os tratamentos com a aplicação de corretivos não houve diferença significativa (Tabela 4). Ao contrário do observado neste trabalho, Sousa et al. (2012) observaram um melhor desempenho como resultado da aplicação de enxofre elementar, e de gesso agrícola em solo salinosódico, sobre a produção de matéria seca de espécies arbóreas do bioma Caatinga. Contudo, essa diferença pode estar associada com as diferenças entre as espécies vegetais e com os níveis de salinidade estabelecidos pelos respectivos tratamentos, após a aplicação dos corretivos. Comparando-se as espécies vegetais, à exceção do pereiro, independentemente dos tratamentos de correção do solo, praticamente não foram observadas diferenças nas respectivas produções de matéria seca (Tabela 3).

Os desempenhos das espécies vegetais, nos diferentes tratamentos de correção do solo, podem ser mais bem avaliados por meio das respectivas produções de matéria seca total, em relação à do tratamento sem corretivo (MSTrel) (Figura 1). Como ocorreu com a produção de matéria seca absoluta (Tabela 3), em geral, a aplicação dos corretivos proporcionou valores de produção relativa de matéria seca superiores a 100\%, ou seja, superiores a do tratamento sem corretivo, embora a magnitude da resposta tenha variado entre as espécies vegetais. Observou-se que as arbóreas jurema-preta e tamboril apresentaram maior crescimento relativo em matéria seca, sendo as plantas com maior resposta ou tolerância às condições de salinidade estabelecidas pelos tratamentos com correti-

Tabela 3. Crescimento em altura, diâmetro e acúmulo de matéria seca de plantas arbóreas após 90 dias do transplantio, em função dos tratamentos de correção do solo

\begin{tabular}{|c|c|c|c|c|c|}
\hline Tratamentos & Craibeira & Jurema-preta & Pereiro & Sabiá & Tamboril \\
\hline & \multicolumn{5}{|c|}{ Crescimento em altura $(\mathrm{cm})$} \\
\hline SSC & $20,17 \mathrm{aBC}$ & $64,00 \mathrm{bA}$ & $8,17 \mathrm{aC}$ & $48,27 \mathrm{abAB}$ & $31,33 \mathrm{cABC}$ \\
\hline $50 \% \mathrm{NG}+\mathrm{MO}$ & $23,33 \mathrm{aBC}$ & $91,50 \mathrm{abA}$ & $2,67 \mathrm{aC}$ & $44,07 \mathrm{bB}$ & $88,00 \mathrm{aA}$ \\
\hline $100 \% \mathrm{NG}$ & $35,50 \mathrm{aBC}$ & $108,50 \mathrm{aA}$ & $6,83 \mathrm{aC}$ & $66,36 \mathrm{abB}$ & $53,17 \mathrm{bcB}$ \\
\hline $50 \% \mathrm{~S}+\mathrm{MO}$ & $23,83 \mathrm{aB}$ & $100,17 \mathrm{aA}$ & $15,00 \mathrm{aB}$ & $79,33 \mathrm{aA}$ & $97,67 \mathrm{aA}$ \\
\hline \multirow[t]{2}{*}{$100 \% \mathrm{~S}$} & $18,67 \mathrm{aB}$ & $108,50 \mathrm{aA}$ & $7,50 \mathrm{aB}$ & $78,67 \mathrm{aA}$ & $81,83 \mathrm{abA}$ \\
\hline & \multicolumn{5}{|c|}{ Crescimento em diâmetro do caule (mm) } \\
\hline SSC & $4,47 \mathrm{abA}$ & $3,19 \mathrm{aA}$ & $2,05 \mathrm{aA}$ & $3,64 \mathrm{aA}$ & $3,15 \mathrm{bA}$ \\
\hline $50 \% \mathrm{NG}+\mathrm{MO}$ & $5,38 \mathrm{abAB}$ & $5,23 \mathrm{aABC}$ & $2,46 \mathrm{aC}$ & $3,37 \mathrm{aBC}$ & $7,13 \mathrm{aA}$ \\
\hline $100 \% \mathrm{NG}$ & $6,92 \mathrm{aA}$ & $4,68 \mathrm{aAB}$ & $3,01 \mathrm{aB}$ & $4,38 \mathrm{aAB}$ & $5,59 \mathrm{abAB}$ \\
\hline $50 \% \mathrm{~S}+\mathrm{MO}$ & $5,32 \mathrm{abAB}$ & $5,87 \mathrm{aAB}$ & $3,20 \mathrm{aB}$ & $4,72 \mathrm{aAB}$ & $7,59 \mathrm{aA}$ \\
\hline \multirow[t]{2}{*}{$100 \% \mathrm{~S}$} & $3,96 \mathrm{bAB}$ & $5,13 \mathrm{aAB}$ & $3,30 \mathrm{aB}$ & $5,38 \mathrm{aAB}$ & $6,64 \mathrm{aA}$ \\
\hline & \multicolumn{5}{|c|}{ Matéria seca da parte aérea $\left(\mathrm{g}\right.$ vaso $\left.{ }^{-1}\right)$} \\
\hline SSC & $15,15 \mathrm{aA}$ & $10,05 \mathrm{bA}$ & $3,57 \mathrm{aB}$ & $14,25 \mathrm{bA}$ & $8,91 \mathrm{bA}$ \\
\hline $50 \% \mathrm{NG}+\mathrm{MO}$ & $17,55 \mathrm{aAB}$ & $22,18 \mathrm{aAB}$ & $2,55 \mathrm{aC}$ & $13,82 \mathrm{bB}$ & $29,36 \mathrm{aA}$ \\
\hline $100 \% \mathrm{NG}$ & $23,09 \mathrm{aA}$ & $22,98 \mathrm{aA}$ & $3,65 \mathrm{aB}$ & $23,95 \mathrm{abA}$ & $19,16 \mathrm{abA}$ \\
\hline $50 \% \mathrm{~S}+\mathrm{MO}$ & $17,84 \mathrm{aA}$ & $23,01 \mathrm{aA}$ & $5,43 \mathrm{aB}$ & $23,62 \mathrm{abA}$ & $29,40 \mathrm{aA}$ \\
\hline \multirow[t]{2}{*}{$100 \% \mathrm{~S}$} & $15,09 \mathrm{aBC}$ & $23,73 \mathrm{aAB}$ & $5,16 \mathrm{aC}$ & $28,34 \mathrm{aA}$ & $26,08 \mathrm{aAB}$ \\
\hline & \multicolumn{5}{|c|}{ Matéria seca de raízes $\left(\mathrm{g} \mathrm{vaso}^{-1}\right.$ ) } \\
\hline SSC & $4,38 \mathrm{aA}$ & $2,89 \mathrm{bA}$ & $1,03 \mathrm{aC}$ & $3,91 \mathrm{aA}$ & $2,25 \mathrm{bA}$ \\
\hline $50 \% \mathrm{NG}+\mathrm{M} . \mathrm{O}$ & $4,71 \mathrm{aBC}$ & $10,15 \mathrm{aA}$ & $1,37 \mathrm{aC}$ & $2,40 \mathrm{aBC}$ & 7,04 abAB \\
\hline $100 \% \mathrm{NG}$ & $7,92 \mathrm{aA}$ & $8,82 \mathrm{aA}$ & $1,63 \mathrm{aB}$ & $5,89 \mathrm{aAB}$ & $8,17 \mathrm{aA}$ \\
\hline $50 \% \mathrm{~S}+\mathrm{M} . \mathrm{O}$ & $5,32 \mathrm{aA}$ & $5,65 \mathrm{abA}$ & $1,18 \mathrm{aC}$ & $3,25 \mathrm{aA}$ & $5,40 \mathrm{abA}$ \\
\hline \multirow[t]{2}{*}{$100 \% \mathrm{~S}$} & $4,69 \mathrm{aA}$ & $7,20 \mathrm{abA}$ & $2,07 \mathrm{aB}$ & 5,17 aA & $6,50 \mathrm{abA}$ \\
\hline & \multicolumn{5}{|c|}{ Matéria seca total $\left(\mathrm{g} \mathrm{vaso}^{-1}\right)$} \\
\hline SSC & $19,53 \mathrm{aA}$ & $12,95 \mathrm{bA}$ & 4,60 aA & $18,16 \mathrm{abA}$ & $11,17 \mathrm{bA}$ \\
\hline $50 \% \mathrm{NG}+\mathrm{M} . \mathrm{O}$ & $22,26 \mathrm{aAB}$ & $32,27 \mathrm{aA}$ & $3,92 \mathrm{aC}$ & $16,22 \mathrm{bB}$ & $36,40 \mathrm{aA}$ \\
\hline $100 \% \mathrm{NG}$ & $31,01 \mathrm{aA}$ & $31,80 \mathrm{aA}$ & $5,28 \mathrm{aB}$ & $29,84 \mathrm{abA}$ & $27,33 \mathrm{aA}$ \\
\hline $50 \% \mathrm{~S}+\mathrm{M} . \mathrm{O}$ & $23,17 \mathrm{aA}$ & $28,66 \mathrm{abA}$ & $6,61 \mathrm{aB}$ & $26,87 \mathrm{abA}$ & $34,80 \mathrm{aA}$ \\
\hline $100 \% \mathrm{~S}$ & $19,79 \mathrm{aAB}$ & $30,93 \mathrm{aA}$ & $7,25 \mathrm{aB}$ & $33,50 \mathrm{aA}$ & $32,58 \mathrm{aA}$ \\
\hline
\end{tabular}

SSC: Solo sem corretivo; NG: Necessidade de gesso; MO: Matéria orgânica na dose de 15\% (v/v); S: enxofre elementar. Médias seguidas de letras iguais, minúsculas nas colunas e maiúsculas nas linhas, não diferem entre si (Tukey a 5\%). 
Tabela 4. Variáveis relacionadas com as trocas gasosas de plantas arbóreas, em função dos tratamentos de correção do solo

\begin{tabular}{|c|c|c|c|c|c|}
\hline Tratamentos & Craibeira & Jurema-preta & Pereiro & Sabiá & Tamboril \\
\hline & \multicolumn{5}{|c|}{ Taxa fotossintética $\left(\mu \mathrm{mol} \mathrm{m}{ }^{-2} \mathrm{~s}^{-1}\right)$} \\
\hline SSC & $4,25 \mathrm{bC}$ & $9,33 \mathrm{cAB}$ & $5,28 \mathrm{bBC}$ & $8,35 \mathrm{bABC}$ & $10,35 \mathrm{aA}$ \\
\hline $50 \% \mathrm{NG}+\mathrm{MO}$ & $13,65 \mathrm{aA}$ & $14,82 \mathrm{bA}$ & $3,76 \mathrm{bB}$ & $10,62 \mathrm{bA}$ & $12,06 \mathrm{aA}$ \\
\hline $100 \% \mathrm{NG}$ & $11,07 \mathrm{aB}$ & $19,31 \mathrm{abA}$ & $7,26 \mathrm{aB}$ & $9,59 \mathrm{bB}$ & $7,71 \mathrm{aB}$ \\
\hline $50 \% \mathrm{~S}+\mathrm{M} . \mathrm{O}$ & $11,76 \mathrm{aB}$ & $17,27 \mathrm{abA}$ & $3,76 \mathrm{bC}$ & $9,83 \mathrm{bB}$ & $9,35 \mathrm{aB}$ \\
\hline \multirow[t]{2}{*}{$100 \% \mathrm{~S}$} & $11,05 \mathrm{aB}$ & $19,76 \mathrm{aA}$ & $7,70 \mathrm{aB}$ & $16,44 \mathrm{aA}$ & $11,18 \mathrm{aB}$ \\
\hline & \multicolumn{5}{|c|}{ Taxa de transpiração $\left(\mathrm{mmol} \mathrm{m}^{-2} \mathrm{~s}^{-1}\right)$} \\
\hline SSC & $1,99 \mathrm{bB}$ & $2,34 \mathrm{bB}$ & $1,63 \mathrm{aB}$ & $3,03 \mathrm{abA}$ & $2,28 \mathrm{aB}$ \\
\hline $50 \% \mathrm{NG}+\mathrm{MO}$ & $2,88 \mathrm{aAB}$ & $4,00 \mathrm{aA}$ & $1,20 \mathrm{aC}$ & $1,94 \mathrm{bBC}$ & $2,81 \mathrm{aAB}$ \\
\hline $100 \% \mathrm{NG}$ & $3,26 \mathrm{aAB}$ & $3,68 \mathrm{abA}$ & $1,48 \mathrm{aC}$ & 2,32 abABC & $1,90 \mathrm{aBC}$ \\
\hline $50 \% \mathrm{~S}+\mathrm{MO}$ & $3,21 \mathrm{aA}$ & $3,15 \mathrm{abA}$ & $1,60 \mathrm{aB}$ & $2,74 \mathrm{abAB}$ & $2,67 \mathrm{aAB}$ \\
\hline \multirow[t]{2}{*}{$100 \% \mathrm{~S}$} & $2,63 \mathrm{aABC}$ & 4,06 aA & $1,84 \mathrm{aC}$ & $3,69 \mathrm{aAB}$ & $2,44 \mathrm{aBC}$ \\
\hline & \multicolumn{5}{|c|}{ Condutância estomática $\left(\mathrm{mol} \mathrm{m}^{-2} \mathrm{~s}^{-1}\right)$} \\
\hline SSC & $0,10 \mathrm{bA}$ & $0,15 \mathrm{bA}$ & $0,08 \mathrm{aA}$ & $0,17 \mathrm{abA}$ & $0,19 \mathrm{aA}$ \\
\hline $50 \% \mathrm{NG}+\mathrm{MO}$ & $0,19 \mathrm{aAB}$ & $0,26 \mathrm{abA}$ & $0,06 \mathrm{aB}$ & $0,18 \mathrm{abAB}$ & $0,25 \mathrm{aA}$ \\
\hline $100 \% \mathrm{NG}$ & $0,19 \mathrm{aB}$ & $0,35 \mathrm{aA}$ & $0,08 \mathrm{aB}$ & $0,14 \mathrm{bB}$ & $0,14 \mathrm{aB}$ \\
\hline $50 \% \mathrm{~S}+\mathrm{MO}$ & $0,21 \mathrm{aAB}$ & $0,32 \mathrm{aA}$ & $0,09 \mathrm{aB}$ & $0,28 \mathrm{abA}$ & $0,18 \mathrm{aAB}$ \\
\hline \multirow[t]{2}{*}{$100 \% \mathrm{~S}$} & $0,19 \mathrm{aAB}$ & $0,31 \mathrm{aA}$ & $0,11 \mathrm{aB}$ & $0,30 \mathrm{aA}$ & $0,22 \mathrm{aAB}$ \\
\hline & \multicolumn{5}{|c|}{ Concentração intercelular de $\mathrm{CO}_{2}\left(\mathrm{mg} \mathrm{L}^{-1}\right)$} \\
\hline SSC & $230,0 \mathrm{aA}$ & $224,5 \mathrm{aA}$ & $252,0 \mathrm{aA}$ & $221,0 \mathrm{aA}$ & $268,5 \mathrm{aA}$ \\
\hline $50 \% \mathrm{NG}+\mathrm{MO}$ & $222,0 \mathrm{aA}$ & $214,0 \mathrm{aA}$ & $257,3 \mathrm{aA}$ & $254,8 \mathrm{aA}$ & $220,2 \mathrm{aA}$ \\
\hline $100 \% \mathrm{NG}$ & $241,5 \mathrm{aA}$ & $224,5 \mathrm{aA}$ & $232,5 \mathrm{aA}$ & $229,3 \mathrm{aA}$ & $250,3 \mathrm{aA}$ \\
\hline $50 \% \mathrm{~S}+\mathrm{MO}$ & $242,5 \mathrm{aA}$ & $253,3 \mathrm{aA}$ & $256,7 \mathrm{aA}$ & $251,0 \mathrm{aA}$ & $239,8 \mathrm{aA}$ \\
\hline $100 \% \mathrm{~S}$ & $249,5 \mathrm{aA}$ & $214,3 \mathrm{aA}$ & $244,7 \mathrm{aA}$ & $217,5 \mathrm{aA}$ & $260,7 \mathrm{aA}$ \\
\hline
\end{tabular}

SSC: Solo sem corretivo; NG: Necessidade de gesso; MO: Matéria orgânica na dose de 15\% (v/v); S: enxofre elementar. Médias seguidas de letras iguais, minúsculas nas colunas e maiúsculas nas linhas, não diferem entre si (Tukey a 5\%).

vos (Figura 1). Assim, essas arbóreas apresentariam maior potencial para serem cultivadas em solos salinizados, tratados com os corretivos avaliados neste trabalho, principalmente tamboril, nos tratamentos 50\% NG + MO, 50\% $\mathrm{S}+\mathrm{MO}$ e $100 \% \mathrm{~S}$.

A maior produção de matéria seca nos tratamentos com a aplicação de corretivos, provavelmente, deve-se ao estabelecimento de melhores condições químicas, em relação às do tratamento controle (Tabela 2), conforme verificado em outros trabalhos (Parida et al., 2004; Stamford et al., 2007; Sousa et al., 2010). Como observado, estes tratamentos proporcionaram diminuição dos valores de pH e PST e aumento dos teores de $\mathrm{Ca}$ e $\mathrm{Mg}$, o que pode ter favorecido o crescimento das plantas.

Em relação às respostas fisiológicas das plantas, observou-se que, para taxa fotossintética, de maneira geral, a arbórea jurema-preta apresentou os maiores valores para esta variável (Tabela 4), assim como ocorreu para o crescimento em altura. A arbórea pereiro, por outro lado, apresentou os menores valores de taxa fotossintética, concordando com seu pequeno incremento em altura e em diâmetro (Tabela 3). Em relação aos tratamentos de correção do solo, verificou-se que, nas arbóreas craibeira e jurema-preta, o solo salino sem corretivo proporcionou diminuição da taxa fotossintética dessas plantas, em relação aos demais tratamentos. Nas demais arbóreas, isso não ocorreu, embora, para pereiro e sabiá, os maiores valores para essa variável tenham sido obtidos com a aplicação de enxofre elementar, na dose correspondente a $100 \%$ da necessidade de gesso. A taxa de transpiração e a

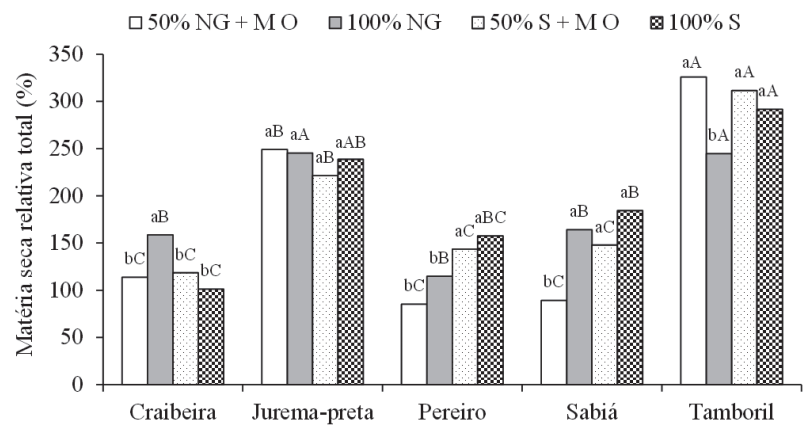

Figura 1. Produção de matéria seca relativa de plantas arbóreas em função dos tratamentos de correção do solo: SSC: Solo sem corretivo; NG: Necessidade de gesso; MO: Matéria orgânica na dose de $15 \%(\mathrm{v} / \mathrm{v})$; S: enxofre elementar. Médias seguidas de letras iguais minúsculas comparando os tratamentos de correção do solo, e maiúsculas comparando as espécies arbóreas, não diferem entre si (Tukey a 5\%). 
Tabela 5. Coeficientes de correlação simples de Pearson (r) entre atributos químicos do solo e variáveis fisiológicas

\begin{tabular}{|c|c|c|c|c|c|c|}
\hline Variáveis fisiológicas & pH & CEes & $\mathrm{Na}^{+}$ & $\mathbf{C a}^{+2}$ & PST & $\mathbf{K}^{+}$ \\
\hline \multicolumn{7}{|c|}{ Jurema-preta } \\
\hline $\mathrm{Ci}$ & $0,11 \mathrm{~ns}$ & $-0,13 \mathrm{~ns}$ & $-0,10 \mathrm{~ns}$ & $-0,17 \mathrm{~ns}$ & $0,05 \mathrm{~ns}$ & $0,24 \mathrm{~ns}$ \\
\hline $\mathrm{E}$ & $-0,70 * *$ & $0,73 * *$ & $-0,22 \mathrm{~ns}$ & $0,44^{*}$ & $-0,52 *$ & $0,21 \mathrm{~ns}$ \\
\hline GS & $-0,64 * *$ & $0,34 \mathrm{~ns}$ & $-0,17 \mathrm{~ns}$ & $0,51 *$ & $-0,70 *$ & $0,21 \mathrm{~ns}$ \\
\hline A & $-0,87 * *$ & $0,50 *$ & $-0,22 \mathrm{~ns}$ & $0,56^{*}$ & $-0,76 * *$ & $0,17 \mathrm{~ns}$ \\
\hline \multicolumn{7}{|c|}{ Craibeira } \\
\hline $\mathrm{Ci}$ & $-0,34 \mathrm{~ns}$ & $0,08 \mathrm{~ns}$ & $0,06 \mathrm{~ns}$ & $0,11 \mathrm{~ns}$ & $-0,17 \mathrm{nsa}$ & $-0,10 \mathrm{~ns}$ \\
\hline $\mathrm{E}$ & $-0,47 *$ & $0,01 \mathrm{~ns}$ & $-0,41^{*}$ & $0,35 \mathrm{~ns}$ & $-0,54^{*}$ & $0,31 \mathrm{~ns}$ \\
\hline Gs & $-0,55^{*}$ & $0,16 \mathrm{~ns}$ & $-0,27 \mathrm{~ns}$ & $0,27 \mathrm{~ns}$ & $-0,48 *$ & $0,28 \mathrm{~ns}$ \\
\hline A & $-0,74 *$ & $0,69 *$ & $-0,32 \mathrm{~ns}$ & $0,46^{*}$ & $-0,67 *$ & $0,61 * *$ \\
\hline \multicolumn{7}{|c|}{ Pereiro } \\
\hline $\mathrm{Ci}$ & $0,20 \mathrm{~ns}$ & $-0,03 \mathrm{~ns}$ & $0,14 \mathrm{~ns}$ & $-0,21 \mathrm{~ns}$ & $0,20 \mathrm{~ns}$ & $0,29 \mathrm{~ns}$ \\
\hline $\mathrm{E}$ & $-0,10 \mathrm{~ns}$ & $-0,26 \mathrm{~ns}$ & $0,04 \mathrm{~ns}$ & $-0,25 \mathrm{~ns}$ & $0,09 \mathrm{~ns}$ & $-0,18 \mathrm{~ns}$ \\
\hline Gs & $-0,26 \mathrm{~ns}$ & $-0,04 \mathrm{~ns}$ & $0,15 \mathrm{~ns}$ & $-0,11 \mathrm{~ns}$ & $0,02 \mathrm{~ns}$ & $-0,14 \mathrm{~ns}$ \\
\hline A & $-0,37 \mathrm{~ns}$ & $-0,03 \mathrm{~ns}$ & $-0,11 \mathrm{~ns}$ & $0,25 \mathrm{~ns}$ & $-0,19 \mathrm{~ns}$ & $-0,55^{*}$ \\
\hline \multicolumn{7}{|c|}{ Sabiá } \\
\hline $\mathrm{Ci}$ & $0,04 \mathrm{~ns}$ & $0,20 \mathrm{~ns}$ & $0,00 \mathrm{~ns}$ & $0,03 \mathrm{~ns}$ & $-0,09 \mathrm{~ns}$ & $0,70 * *$ \\
\hline $\mathrm{E}$ & $-0,03 n s$ & $-0,21 \mathrm{~ns}$ & $0,18 \mathrm{~ns}$ & $-0,37 \mathrm{~ns}$ & $0,17 \mathrm{~ns}$ & $-0,20 \mathrm{~ns}$ \\
\hline Gs & $-0,28 \mathrm{~ns}$ & $0,09 \mathrm{~ns}$ & $0,21 \mathrm{~ns}$ & $-0,42 \mathrm{~ns}$ & $-0,01 \mathrm{~ns}$ & $0,41 \mathrm{~ns}$ \\
\hline A & $-0,49 *$ & $0,38 \mathrm{~ns}$ & $-0,19 \mathrm{~ns}$ & $-0,03 \mathrm{~ns}$ & $-0,21 \mathrm{~ns}$ & $0,02 \mathrm{~ns}$ \\
\hline \multicolumn{7}{|c|}{ Tamboril } \\
\hline $\mathrm{Ci}$ & $0,23 \mathrm{~ns}$ & $-0,51 *$ & $0,09 \mathrm{~ns}$ & $-0,19 \mathrm{~ns}$ & $0,16 \mathrm{~ns}$ & $-0,38 \mathrm{~ns}$ \\
\hline E & $0,15 \mathrm{~ns}$ & $0,15 \mathrm{~ns}$ & $0,08 \mathrm{~ns}$ & $-0,36 \mathrm{~ns}$ & $0,18 \mathrm{~ns}$ & $0,47 *$ \\
\hline Gs & $0,08 \mathrm{~ns}$ & $-0,05 \mathrm{~ns}$ & $0,03 \mathrm{~ns}$ & $-0,23 \mathrm{~ns}$ & $0,19 \mathrm{~ns}$ & $0,19 \mathrm{~ns}$ \\
\hline A & $0,20 \mathrm{~ns}$ & $0,09 \mathrm{~ns}$ & $0,34 \mathrm{~ns}$ & $-0,37 \mathrm{~ns}$ & $0,31 \mathrm{~ns}$ & $0,29 \mathrm{~ns}$ \\
\hline
\end{tabular}

$\mathrm{Ci}$ = concentração intercelular de $\mathrm{CO}_{2} ; \mathrm{E}=$ taxa de transpiração; Gs = condutância estomática; $\mathrm{A}=$ taxa fotossintética. PST: percentagem de sódio trocável; ns, * e **: não significativo, significativo a 5 e a $1 \%$, respectivamente, pelo teste t.

condutância estomática, de maneira geral, apresentaram respostas aos tratamentos semelhantes à taxa fotossintética. Da mesma forma, pereiro apresentou, em geral, os menores valores para ambas as variáveis e, jurema-preta, os maiores. Em relação aos tratamentos de correção do solo, verificou-se que, a exemplo do que se viu em relação à taxa fotossintética, o tratamento sem corretivo proporcionou diminuição dos valores dessas variáveis para as arbóreas craibeira e jurema-preta, em relação aos demais tratamentos. Para o tamboril, assim como ocorreu para a taxa fotossintética, não houve influência dos tratamentos sobre a taxa de transpiração e sobre a condutância estomática (Tabela 4).

Correlacionando-se os valores das variáveis fisiológicas com alguns atributos do solo, verificaram-se poucas correlações significativas (Tabela 5). Contudo, observouse, especialmente nas arbóreas jurema-preta e craibeira, correlação negativa entre os valores de taxa fotossintética, taxa de transpiração e condutância estomática e os valores de pH e PST do solo. Esses resultados estão de acordo com os observados em outros trabalhos (Sousa et al., 2010; Sousa et al., 2012). Isso ocorre porque o estresse salino provoca diminuição do potencial de turgescência dos tecidos foliares (Taiz \& Zeiger, 2004), provocando o fechamento dos estômatos, o aumento da resistência à difusão do $\mathrm{CO}_{2}$ e a diminuição da taxa fotossintética (Romero-Aranda et al., 1998; Chaves et al., 2009).

\section{CONCLUSÕES}

A aplicação de gesso agrícola ou enxofre elementar proporcionou melhorias químicas no solo salino-sódico estudado, especialmente diminuindo a percentagem de sódio trocável e aumentando os teores de cálcio e magnésio trocáveis.

A aplicação de gesso agrícola ou enxofre elementar, com ou sem matéria orgânica, proporcionou incrementos no crescimento e acúmulo de massa de matéria seca das espécies arbóreas, especialmente jurema-preta (Mimosa tenuiflora), sabiá (Mimosa caelsalpiniifolia) e tamboril (Enterolobium contortisiliquum). Esses tratamentos diminuíram os efeitos estressantes do solo salino-sódico sobre as plantas, principalmente sobre a taxa fotossintética. 


\section{REFERÊNCIAS}

Chaves MM, Flexas J \& Pinheiro C (2009) Photosynthesis under drought and salt stress: regulation mechanisms from whole plant to cell. Annals of Botany, 103:551-560.

Duran R, Garcia J \& Amaya R (2000) Amendment evaluation for the reclamation of sodic soils in the Cesar Valley. Suelos Ecuatoriales, 30:21-28.

El-Dardiry EI (2007) Effect of soil and water salinity on barley grains germination under some amendments. Word Journal of Agricultural Sciences, 3:329-338.

Embrapa (1997) Manual de métodos de análise de solo. $2^{\text {a }}$ ed. Rio de Janeiro, Centro Nacional de Pesquisa de Solos. 212p.

Freitas RMO, Nogueira NW, Oliveira FN, Costa EM \& Ribeiro MCC (2010) Efeito da irrigação com água salina na emergência e crescimento inicial de plântulas de jucá. Revista Caatinga, 23:54-58.

Gharaibeh MA, Eltaif NI \& Shunnar OF (2009) Leaching and reclamation of calcareous saline-sodic soil by moderately saline and moderate-SAR water using gypsum and calcium chloride. Journal Plant Nutrition Soil Science, 172:713-719.

Gill JS, Sale PWG \& Tang C (2008) Amelioration of dense sodic subsoil using organic amendments increases wheat yield more than using gypsum in a high rainfall zone of southern Australia. Field Crops Research, 107:265-275.

Gill JS, Sale PWG, Peries RR \& Tang C (2009) Changes in soil physical properties and crop root growth in dense sodic subsoil following incorporation of organic amendments Field Crops Research, 114:137-146.

Hasegawa PM, Bressan RA, Zhu JK \& Bohnert HJ (2000) Plant cellular and molecular responses to high salinity. Annual Review of Plant Physiology and Plant Molecular Biology, 51:463-499.

Holanda AC, Santos RV, Souto JS \& Alves AR (2007) Desenvolvimento inicial de espécies arbóreas em ambientes degradados por sais. Revista de Biologia e Ciência da Terra, 7:39-50.

Leal IG, Accioly AMA, Nascimento CWA, Freire MBGS, Montenegro AAA \& Ferreira FL (2008) Fitorremediação de solo salino sódico por Atriplex nummularia e gesso de jazida. Revista Brasileira de Ciência do Solo, 32:1065-1072.

Leite EM, Cavalcante LFC, Diniz AA, Santos RV, Alves GSL \& Cavalcante IHL (2007) Correção da sodicidade de dois solos irrigados em resposta à aplicação de gesso agrícola. Irriga, 12:168-176.

McBride MB (1995) Environmental chemistry of soils. New York, Oxford University Press. 416p.

Melo RM, Barros, MFC, Santos PM \& Rolim MM (2008) Correção de solos salino-sódicos pela aplicação de gesso mineral. Revista Brasileira de Engenharia Agrícola e Ambiental, 12:376-380.

Mohamed AI, Ali OM \& Matloub MA (2007) Effect of soil amendments on some physical and chemical properties of some soils of Egypt under saline irrigation water. African Crop Science Conference Proceedings. 8:1571-1578.

Munns R (2002) Comparative physiology of salt and water stress. Plant, Cell and Environment, 25:239-250.

Parida AK, Das AB \& Mittra B (2004) Effects of salt on growth, ion accumulation photosynthesis and leaf anatomy of the mangrove, Bruguiera parviflora. Trees - Structure and Function, 18:167-174.

Pazhanivelan S, Amanullah MM, Vaiyapuri K, Athyamoorthi K \& Radhamani S (2008) Influence of planting techniques and amendments on the performance of tamarind (Tamarindus indicus) and changes in soil properties in rainfed alkali soil. Research Journal of Agriculture and Biological Sciences, 4:285-288.
Qadir M, Oster JD, Schubert S, Noble AD \& Sahrawat KL (2007) Phytoremediation of sodic and saline-sodic soils. Advances in Agronomy, 96:197-247.

Qadir M, Qureshi RH \& Ahmad N (1996) Reclamation of a salinesodic soil by gypsum and Leptochloa fusca. Geoderma, 74:207217.

Raij B van (1991) Fertilidade do solo e adubação. Campinas, Ceres. 346 p.

Ribeiro MR, Freire FJ \& Montenegro AAA (2003) Solos halomórficos do Brasil: Ocorrência, gênese, classificação, uso e manejo sustentável. In: Curi N, Marques JJ, Guilherme LRG, Lima JM, Lopes AS \& Alvarez VH (Eds.) Tópicos em ciência do solo. Viçosa, Sociedade Brasileira de Ciência do Solo. p.165208 .

Richards LA (1954) Diagnosis and improvement of saline and alkali soils. Washington, Department of Agriculture, USDA Agricultural Handbook 60.160p.

Romero-Aranda R, Moya JL, Tadeo FR, Lagaz F, Primo-Millo E \& Talon M (1998) Physiological and anatomical disturbances induced by chloride salts in sensitive and tolerant citrus: Beneficial and detrimental effects of cations. Plant, Cell and Environment, 21:1243-1253.

Santos RV \& Muraoka T (1997) Interação salinidade e fertilidade do solo. In: Gheyi HR, Queiroz JE \& Medeiros JF (Eds.) Manejo e controle da salinidade na agricultura irrigada. Campina Grande, UFPB. p.289-317.

Sousa FQ, Araujo JL, Pereira FHF, Nascimento MGR, Lima GS, Pereira EB \& Santos RV (2010) Crescimento de espécies arbóreas nativas do semiárido cultivadas em um solo degradado pelo excesso de sais e de sódio tratado com corretivos. In: Reunião brasileira de fertilidade do solo e nutrição de plantas (Fertbio), Guarapari. Anais, SBCS/ CD-ROM.

Sousa FQ, Araújo JL, Silva AP, Pereira FHF, Santos RV \& Lima GS (2012) Crescimento e respostas fisiológicas de espécies arbóreas em solo salinizado tratado com corretivos. Revista Brasileira de Engenharia Agrícola e Ambiental, 6:173-181.

Stamford NP, Freitas ADS, Ferraz DS \& Santos CES (2002) Effect of sulphur inoculated with Thiobacillus on saline soils amendment and growth of cowpea and yam bean legumes. Journal of Agricultural Science, 139:275-281.

Stamford NP, Ribeiro MR \& Cunha KPV (2007) Effectiveness of sulfur with Acidithiobacillus and gypsum in chemical attributes of a Brazilian sodic soil. World Journal Microbiology Biotechnology, 23:1433-1439.

Taiz L \& Zeiger E (2004) Fisiologia do estresse. In: Taiz L \& Zeiger E (Eds.) Fisiologia vegetal. 3 ${ }^{\text {a }}$ ed. Califórnia, Artmed. p.613-643.

Tertuliano SSX \& Santos RV (1998) Crescimento de espécies arbóreas em solo salino-sódico tratado com ácido sulfúrico. Revista Brasileira de Engenharia Agrícola e Ambiental, 2:239242 .

Vital AFM, Santos RV, Cavalcante LFC \& Souto JS (2005) Comportamento de atributos químicos de um solo salino-sódico tratado com gesso e fósforo. Revista Brasileira de Engenharia Agrícola e Ambiental, 9:30-36.

Zaka MA, Mujeeb F, Sarwar G, Hassan NM \& Hassan G (2003) Agromelioration of saline-sodic soils. Journal of Biological Sciences, 3:329-334.

Zia MH, Sabir SM, Ghafoor A \& Murtaza G (2007) Effectiveness of sulphuric acid and gypsum for the reclamation of a calcareous saline-sodic soil under four crop rotations. Journal Agronomy \& Crop Science, 193:262-269. 